\title{
Closed-Form Solutions for Gradient Elastic Beams with Geometric Discontinuities by Laplace Transform
}

\author{
Mustafa Özgür Yayli \\ Department of Civil Engineering, Faculty of Engineering, Bilecik Şeyh Edebali University, Bilecik, 11210 Gülümbe, Turkey \\ Correspondence should be addressed to Mustafa Özgür Yayli; ozguryayli@msn.com
}

Received 26 July 2013; Accepted 27 October 2013

Academic Editor: Evangelos J. Sapountzakis

Copyright (c) 2013 Mustafa Özgür Yayli. This is an open access article distributed under the Creative Commons Attribution License, which permits unrestricted use, distribution, and reproduction in any medium, provided the original work is properly cited.

The static bending solution of a gradient elastic beam with external discontinuities is presented by Laplace transform. Its utility lies in the ability to switch differential equations to algebraic forms that are more easily solved. A Laplace transformation is applied to the governing equation which is then solved for the static deflection of the microbeam. The exact static response of the gradient elastic beam with external discontinuities is obtained by applying known initial conditions when the others are derived from boundary conditions. The results are given in a series of figures and compared with their classical counterparts. The main contribution of this paper is to provide a closed-form solution for the static deflection of microbeams under geometric discontinuities.

\section{Introduction}

The microbeams are one of the major structures used widely in the fields of MEMS such as those in actuators [1], microswitches [2], microresonators [3], Atomic Force Microscopes [4], and sensors [5] in which thicknesses and lengths of microbeams are typically on the order of microns and submicrons. A large number of those applications utilized the dynamic mechanical properties of thin films materials for targeted performance specifications such as those vibration atomic force microscopes [6].

Microbeams used in MEMS have the dimensions in order of microns and submicrons. It is experimentally observed that not only does the conventional strain-based mechanics underestimate the stiffness of microscale components such as sensors but also it is unable to justify the size effects appearing in these structures $[7,8]$. Due to the lacking of internal length scale parameters, classical strain-based mechanics theories fail to characterize those size effects phenomenon when the structural size is in micron and submicron scale. However, these size dependences can be successfully modeled by employing higher-order continuum theories, in which constitutive equations introduce additional length scale parameters in addition to conventional material parameters [9, 10]. There have been considerable studies on size effects in problems of vibration, bending, buckling, and torsion of microbeams based on nonlocal elasticity theory [11-13]. Yang et al. [14] have proposed the modified couple stress theory recently and only one length scale parameter is included. After this, the modified couple stress and the strain gradient elasticity theories have been widely applied to static and dynamic analysis of microbeams [9, 15-17].

In this study, the static behavior of gradient elastic beams subjected to geometric discontinuties is theoretically investigated in the Laplace domain. Its utility lies in the ability to switch differential equations to algebraic forms that are more easily solved. The material and microbeam models are assumed to obey the strain gradient theory, as developed in $[18,19]$. A Laplace transformation is applied to the governing equation which is then solved for the static deflection of the microbeam. The solutions obtained are transformed to the time domain using the inverse Laplace transform. Static deflection curve is computed exactly and expressed in closed form.

\section{Review of Laplace Transformation and Gradient Elasticity Theory}

2.1. Laplace Transform Applied to Differential Equations. Laplace transformation is a very useful tool for computation of higher order differantial equations. This transformation 
helps to transform differantial equations into the form of algebraic equations which is easier to manipulate. Let $\Omega(t)$ is a function on the interval $[0, \infty)$. The Laplace transform of $\Omega(t)$ is the function in terms of $s$ as follows:

$$
\mathscr{L}_{t}\{\Omega(t)\}=\int_{0}^{\infty} e^{-s t} \Omega(t) d t
$$

Since the Laplace transform yields a function of $s$, the notation $\mathscr{L}[\Omega(t)]=Y(s)$ is used to denote the Laplace transform of $\Omega(t)$. Laplace transforms can be used to solve initial value problems, by transforming a differantial equation to an algebraic equation. Consider the following initial value problem:

$$
\begin{gathered}
\omega_{n} \frac{d^{n} \Omega}{d t^{n}}+\omega_{n-1} \frac{d^{n-1} \Omega}{d t^{n-1}}+\omega_{n-2} \frac{d^{n-2} \Omega}{d t^{n-2}}+\cdots+\omega_{0} \Omega=\Psi(t) \\
\Omega(0)=\Omega_{0}, \quad \Omega^{\prime}(0)=\Omega_{1} \\
\Omega^{\prime \prime}(0)=\Omega_{2}, \ldots, \Omega^{(n-1)}(0)=\Omega_{(n-1)}
\end{gathered}
$$

in which $\omega_{n}, \omega_{n-1}, \omega_{n-2}, \ldots, \omega_{0}$ denote the constants and (3) represents the initial conditions. Taking the Laplace transforms of each term yields

$$
\begin{aligned}
& \omega_{n} \mathscr{L}\left\{\frac{d^{n} \Omega}{d t^{n}}\right\}+\omega_{n-1} \mathscr{L}\left\{\frac{d^{n-1} \Omega}{d t^{n-1}}\right\} \\
& +\omega_{n-2} \mathscr{L}\left\{\frac{d^{n-2} \Omega}{d t^{n-2}}\right\}+\cdots+\omega_{0} \mathscr{L}\{\Omega\}=\mathscr{L}\{\Psi(t)\} .
\end{aligned}
$$

Using the property of the Laplace transformation of derivatives, one can obtain

$$
\begin{aligned}
\omega_{n} & {\left[s^{n} Y(s)-s^{n-1} \Omega(0)-\cdots-\Omega^{n-1}(0)\right] } \\
& +\omega_{n-1}\left[s^{n-1} Y(s)-s^{n-2} \Omega(0)-\cdots-\Omega^{n-2}(0)\right] \\
& +\cdots+\omega_{0} Y(s)=G(s) .
\end{aligned}
$$

The above algebraic equation can be solved for $Y(s)$ and then take the inverse Laplace transform $\left(\mathscr{L}^{-1}\right)$ to obtain the expression for $\Omega(t)$.

2.2. Strain Gradient Elastic Euler Bernoulli Beam Model. In the present study the following gradient elasticity theory is employed which is suitable for investigating the static deflections of microbeams [18]. Consider

$$
\sigma_{i j}=C_{i j k l}\left(\epsilon_{k l}-\gamma^{2} \epsilon_{k l, m n}\right),
$$

in which $\sigma_{i j}$ and $\epsilon_{i j}$ represent the stress and strain tensors, $C_{i j k l}$ represents the elastic moduli, and $\gamma$ denotes the simply gradient elastic modulus, representing the internal or characteristic length of the material microstructure. It is assumed that the microbeam subjected to a concentrated load, as shown in Figure 1. The potential energy of the beam is defined as

$$
U=\frac{1}{2} \mathrm{EI} \int_{0}^{L}\left\{\left(\frac{d^{2} v}{d z^{2}}\right)^{2}-\gamma^{2}\left(\frac{d^{2} v}{\partial z^{2}} \frac{d^{4} v}{d z^{4}}\right)\right\} d z
$$

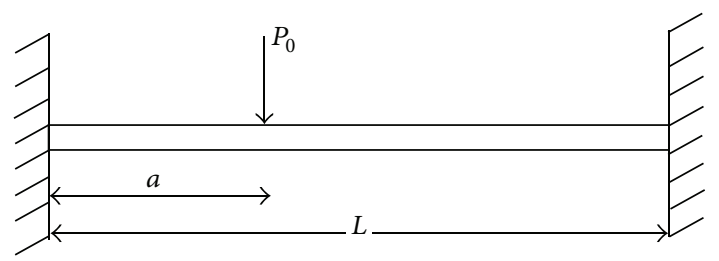

FIGURE 1: Schematic diagram for a microbeam fixed at two ends with concentrated loading.

where EI is the bending stiffness of the microbeam. The variation of the external concentrated load is obtained as:

$$
\varphi W_{E}=\int_{0}^{L} P_{0} \delta(z-a) \varphi v d z
$$

where $P_{0}$ is a concentrated force at an arbitrary point. In order to obtain the governing differantial equation of the system, the above equations substituted into the Hamilton principle resulting in [18]

$$
\mathrm{EI}\left\{\left(\frac{d^{4} v}{d z^{4}}\right)-\gamma^{2}\left(\frac{\partial^{6} v}{\partial z^{6}}\right)\right\}-P_{0} \delta(z-a)=0 .
$$

\subsection{Weak-Form Formulation of the Microbeam Equation and} Nonclassical Boundary Conditions. The starting point for deriving the weak form is to multiply the differential equation of gradient elastic beam with a test function and integrate it over the domain,

$$
\int_{0}^{L} \psi(z)\left(\mathrm{EI}\left\{\left(\frac{d^{4} v}{d z^{4}}\right)-\gamma^{2}\left(\frac{d^{6} v}{d z^{6}}\right)\right\}-P_{0} \delta(z-a)\right) d z=0
$$

where $\psi(z)$ is the test function and is integrating by parts as follows:

$$
\begin{aligned}
0= & \int_{0}^{L}\left(\mathrm{EI}\left[\frac{d^{2} \psi}{d z^{2}} \frac{d^{2} v}{d z^{2}}-\gamma^{2} \frac{d^{3} \psi}{d z^{3}} \frac{d^{3} v}{d z^{3}}\right]\right. \\
& \left.-\psi(z) P_{0} \delta(z-a)\right) d z \\
& +\left\{\psi(z) \mathrm{EI}\left(\frac{d^{3} v}{d z^{3}}-\gamma^{2} \frac{d^{5} v}{d z^{5}}\right)\right\}_{0}^{L} \\
& -\left\{\frac{d \psi(z)}{d z} \mathrm{EI}\left(\frac{d^{2} v}{d z^{2}}-\gamma^{2} \frac{d^{4} v}{d z^{4}}\right)\right\}_{0}^{L} \\
& -\left\{\gamma^{2} \frac{d^{2} \psi(z)}{d z^{2}} \mathrm{EI} \frac{d^{3} v}{d z^{3}}\right\}_{0}^{L} .
\end{aligned}
$$

The presented (11) is a weak derivation of governing equation and the boundary conditions for the microbeam based on gradient elasticity theory. It should be noted that when the scale parameter $\gamma$ is taken to be zero in (11), the gradient elastic beam model decreases to the classical beam model. 
The boundary conditions for the microbeam fixed at two ends (see Figure 1) are

$$
\begin{array}{lll}
\left.v\right|_{z=0}=0, & \left.\frac{\partial v}{\partial z}\right|_{z=0}=0, & \left.\frac{\partial^{3} v}{\partial^{3} z}\right|_{z=0}=0, \\
\left.v\right|_{z=L}=0, & \left.\frac{\partial v}{\partial z}\right|_{z=L}=0, & \left.\frac{\partial^{3} v}{\partial^{3} z}\right|_{z=L}=0 .
\end{array}
$$

\section{Application of Laplace Transform in Gradient Elasticity}

In this section, a Laplace transformation is performed to the governing equation in gradient elasticity which is then solved for the static deflection of the microbeam.

3.1. A Microbeam Subjected to a Concentrated Load. Consider a microbeam subjected to a concentrated load. The concentrated load at $z=a$ can be represented by $P_{0} \delta(z-a)$, where $\delta(\cdot)$ is the Dirac delta function or impulse function. Then the differential equation for the static deflection of the gradient elastic beam is

$$
\mathrm{EI}\left\{\left(\frac{d^{4} v}{d z^{4}}\right)-\gamma^{2}\left(\frac{d^{6} v}{d z^{6}}\right)\right\}=P_{0} \delta(z-a) .
$$

Taking the Laplace transforms of both sides,

$$
\begin{aligned}
& \frac{P_{0}\left(1-e^{-a s}\right)}{\mathrm{EI} s} \\
& =-\gamma^{2}\left(s^{6}\left(\mathscr{L}_{z}[v(z)](s)\right)-v^{(5)}(0)-v^{(4)}(0) s\right. \\
& \left.\quad-v^{(3)}(0) s^{2}-s^{3} v^{\prime \prime}(0)-s^{4} v^{\prime}(0)-v(0) s^{5}\right) \\
& \quad+s^{4}\left(\mathscr{L}_{z}[v(z)](s)\right)-v^{(3)}(0) \\
& \quad-s v^{\prime \prime}(0)-s^{2} v^{\prime}(0)-v(0) s^{3}
\end{aligned}
$$

or

$$
\begin{aligned}
\mathscr{L}_{z}[v(z)](s)= & -\frac{e^{-a s}}{\mathrm{EI} s\left(s^{4}-\gamma^{2} s^{6}\right)} \mathrm{EI} \gamma^{2} v^{(5)}(0) s e^{a s} \\
& +\mathrm{EI}^{2} v^{(4)}(0) s^{2} e^{a s}+\mathrm{EI} \gamma^{2} v^{(3)}(0) s^{3} e^{a s} \\
& -\mathrm{EI} v^{(3)}(0) s e^{a s}+\mathrm{EI} \gamma^{2} s^{4} e^{a s} v^{\prime \prime}(0) \\
& -\mathrm{EI}^{2} e^{a s} v^{\prime \prime}(0)+\mathrm{EI} \gamma^{2} s^{5} e^{a s} v^{\prime}(0) \\
& -\mathrm{EI}^{3} e^{a s} v^{\prime}(0)+\mathrm{EI} \gamma^{2} v(0) s^{6} e^{a s} \\
& -\mathrm{EI} v(0) s^{4} e^{a s}-P_{0} e^{a s}+P_{0} .
\end{aligned}
$$

Using the three known initial conditions in (12),

$$
\begin{aligned}
\mathscr{L}_{z}[v(z)](s)= & \left(-P_{0} e^{-a s}-\mathrm{EI} \gamma^{2} C_{2} s^{2}\right. \\
& \left.+\mathrm{EIC}_{1}\left(s^{2}-\gamma^{2} s^{4}\right)-\mathrm{EI}^{2} C_{3} s+P_{0}\right) \\
& \times\left(\mathrm{EI}\left(s^{5}-\gamma^{2} s^{7}\right)\right)^{-1},
\end{aligned}
$$

where

$$
C_{1}=v^{\prime \prime}(0), \quad C_{2}=v^{(4)}(0), \quad C_{3}=v^{(5)}(0) .
$$

The problem is to find the solution of the differantial equation, as a function of $C_{1}, C_{2}$, and $C_{3}$ and then determine these constants by using the information at the point $z=L$. The inverse Laplace transform $\left(\mathscr{L}^{-1}\right)$ of (17) yields

$$
\begin{aligned}
& \mathscr{L}^{-1}\{v(s)\} \\
& =\mathscr{L}^{-1}\left\{\left(-P_{0} e^{-a s}-\mathrm{EI} \gamma^{2} C_{2} s^{2}+\mathrm{EIC}_{1}\left(s^{2}-\gamma^{2} s^{4}\right)\right.\right. \\
& \left.-\mathrm{EI} \gamma^{2} C_{3} s+P_{0}\right) \\
& \left.\times\left(\mathrm{EI}\left(s^{5}-\gamma^{2} s^{7}\right)\right)^{-1}\right\} \text {, } \\
& v(z)=\frac{1}{\mathrm{EI}}\left(-P_{0} \theta(z-a)\right. \\
& \times\left(-\frac{1}{2} \gamma^{4} e^{(a-z) / \gamma}-\frac{1}{2} \gamma^{4} e^{(z-a) / \gamma}\right. \\
& \left.+\frac{1}{2} \gamma^{2}(a-z)^{2}+\frac{1}{24}(a-z)^{4}+\gamma^{4}\right) \\
& -\frac{1}{6} \gamma^{2} C_{3} \operatorname{EI}\left(z^{3}-3 \gamma^{3} e^{-z / \gamma}\left(e^{2 z / \gamma}-1\right)+6 \gamma^{2} z\right) \\
& -\frac{1}{2} \gamma^{2} C_{2} \text { EI } \\
& \times\left(z^{2}-\gamma^{2} e^{-z / \gamma}\left(e^{z / \gamma}-1\right)^{2}\right) \\
& +\frac{1}{2} C_{1} \operatorname{EI}\left(z^{2}-\gamma^{2} e^{-z / \gamma}\left(e^{z / \gamma}-1\right)^{2}\right) \\
& +\frac{1}{2} \gamma^{2} C_{1} \operatorname{EI}^{-z / \gamma}\left(e^{z / \gamma}-1\right)^{2} \\
& +\frac{1}{24} P_{0}\left(z^{4}+12 \gamma^{2} t^{2}\right. \\
& \left.\left.-12 \gamma^{4} e^{-z / \gamma}\left(e^{z / \gamma}-1\right)^{2}\right)\right),
\end{aligned}
$$

where $\theta(\cdot)$ is the Heaviside step function. Note that the Heaviside step function in (20) turns on at the right edge $\left(z=a^{+}\right)$and the Dirac delta function turns on and off at the same point so we can write the above equation in two parts. For $0 \leq z \leq a$,

$$
\begin{aligned}
v(z)^{\{-a\}} & \\
=\frac{1}{\mathrm{EI}} C_{1}( & \frac{1}{2} \mathrm{EI}\left(z^{2}-\gamma^{2} e^{-z / \gamma}\left(e^{z / \gamma}-1\right)^{2}\right) \\
& \left.+\frac{1}{2} \gamma^{2} \mathrm{EI}^{-z / \gamma}\left(e^{z / \gamma}-1\right)^{2}\right)
\end{aligned}
$$




$$
\begin{aligned}
& -\frac{1}{6} \gamma^{2} C_{3}\left(z^{3}-3 \gamma^{3} e^{-z / \gamma}\left(e^{2 z / \gamma}-1\right)+6 \gamma^{2} z\right) \\
& -\frac{1}{2} \gamma^{2} C_{2}\left(z^{2}-\gamma^{2} e^{-z / \gamma}\left(e^{z / \gamma}-1\right)^{2}\right)
\end{aligned}
$$

and for $a \leq z \leq L$,

$$
\begin{aligned}
& v(z)^{\{+a\}} \\
& =\frac{1}{\mathrm{EI}} C_{1}\left(\frac{1}{2} \mathrm{EI}\left(z^{2}-\gamma^{2} e^{-z / \gamma}\left(e^{z / \gamma}-1\right)^{2}\right)\right. \\
& \left.+\frac{1}{2} \gamma^{2} \mathrm{EI} e^{-z / \gamma}\left(e^{z / \gamma}-1\right)^{2}\right) \\
& +\frac{1}{6} \gamma^{2} C_{3}\left(z^{3}-3 \gamma^{3} e^{-z / \gamma}\left(e^{2 z / \gamma}-1\right)\right. \\
& \left.+6 \gamma^{2} z\right)-\frac{1}{2} \gamma^{2} C_{2} \\
& \times\left(z^{2}-\gamma^{2} e^{-z / \gamma}\left(e^{z / \gamma}-1\right)^{2}\right) \\
& +\frac{1}{\mathrm{EI}} P_{0}\left(-\frac{1}{2} \gamma^{4} e^{(a-z) / \gamma}-\frac{1}{2} \gamma^{4} e^{(z-a) / \gamma}\right. \\
& \left.\quad+\frac{1}{2} \gamma^{2}(a-z)^{2}+\frac{1}{24}(a-z)^{4}+\gamma^{4}\right) .
\end{aligned}
$$

$C_{1}, C_{2}$, and $C_{3}$ are constants to be calculated with the aid of other boundary conditions and (22). For simplicity, the following quantities are defined:

$$
\begin{gathered}
\eta_{1}=e^{L / \gamma} ; \quad \eta_{2}=\eta_{1}^{2}-1 ; \\
\eta_{3}=\eta_{1}+1 ; \quad \eta_{4}=\eta_{1}-1 ; \\
\alpha_{1}=12 \gamma^{3}-L^{3}+6 \gamma^{2} L ; \\
\alpha_{2}=24 \gamma^{4}+L^{4}-8 \gamma^{2} L^{2} ; \\
\alpha_{3}=-144 \gamma^{5}+L^{5}+6 \gamma L^{4}-12 \gamma^{2} L^{3} \\
-96 \gamma^{3} L^{2}-216 \gamma^{4} L ; \\
\alpha_{4}=144 \gamma^{5}+L^{5}-6 \gamma L^{4}-12 \gamma^{2} L^{3} \\
+96 \gamma^{3} L^{2}-216 \gamma^{4} L ; \\
\alpha_{5}=12 \gamma^{3}+L^{3}-6 \gamma^{2} L ; \\
\beta_{1}=-12 \gamma^{3}+L^{3}-12 \gamma^{2} L ; \\
\beta_{2}=-24 \gamma^{4}+L^{4}-12 \gamma^{2} L^{2}-24 \gamma^{3} L ; \\
\beta_{3}=-24 \gamma^{4}+L^{4}-12 \gamma^{2} L^{2}+24 \gamma^{3} L ; \\
\beta_{4}=24 \gamma^{4}+L^{4}-12 \gamma^{2} L^{2} ; \\
\beta_{5}=12 \gamma^{3}+L^{3}-12 \gamma^{2} L
\end{gathered}
$$

By using (23), $C_{1}, C_{2}$, and $C_{3}$ parameters can be calculated as follows:

$$
\begin{aligned}
C_{1}=- & \frac{1}{12 \mathrm{EI} L\left(24 \gamma^{3} \eta_{4}^{2}+\eta_{2} L^{3}-12 \gamma^{2} \eta_{2} L\right)} \\
\times\left(P_{0} e^{-a / \gamma}\right. & \times\left(3 a^{4}\left(\eta_{4}\right) L e^{a / \gamma}\right. \\
& \times\left(-2 \gamma \eta_{1}+2 \gamma+\eta_{1} L+L\right)-4 a^{3} e^{a / \gamma} \\
& \times\left(12 \gamma^{3} \eta_{4}^{2}+2 \eta_{2} L^{3}-3 \gamma \eta_{4}^{2} L^{2}-6 \gamma^{2} \eta_{2} L\right) \\
& +6 a^{2} L e^{a / \gamma}\left(12 \gamma^{3} \eta_{4}^{2}+\eta_{2} L^{3}-6 \gamma^{2} \eta_{2} L\right) \\
& -12 a \gamma L e^{a / \gamma} \\
& \times\left(12 \gamma^{3} \eta_{2}+\left(\eta_{1}^{2}+1\right) L^{3}-6 \gamma^{2} \eta_{3}^{2} L\right) \\
+ & L\left(-12 \alpha_{1} \gamma^{2} e^{2 a / \gamma}+\alpha_{3} e^{a / \gamma}\right. \\
& \quad-12 \alpha_{5} \gamma^{2} \eta_{1}^{2}+12 \alpha_{2} \gamma e^{(a+L) / \gamma} \\
& \quad-\alpha_{4} e^{(a+2 L) / \gamma}+72 \gamma^{4}(2 \gamma-L) \\
& \left.\left.\left.\times e^{(2 a+L) / \gamma}+72 \gamma^{4} \eta_{1}(2 \gamma+L)\right)\right)\right),
\end{aligned}
$$$$
C_{2}=-\frac{1}{2 \gamma \mathrm{EI}\left(24 \gamma^{3} \eta_{4}^{2}+\eta_{2} L^{3}-12 \gamma^{2} \eta_{2} L\right)}
$$$$
\times\left(P_{0} e^{-a / \gamma}\right.
$$$$
\times\left(a^{4} \eta_{4}^{2}\left(-e^{a / \gamma}\right)+2 a^{3} \eta_{4}^{2} L e^{a / \gamma}\right.
$$$$
-12 a^{2} \gamma^{2} \eta_{4}^{2} e^{a / \gamma}+2 \beta_{1} \gamma e^{2 a / \gamma}
$$$$
+\beta_{2} e^{a / \gamma}-2 a e^{a / \gamma}
$$$$
\times\left(12 \gamma^{3} \eta_{2}+\left(\eta_{1}^{2}+1\right) L^{3}\right.
$$$$
\left.-12 \gamma^{2}\left(\eta_{1}^{2}+1\right) L\right)+\beta_{3} e^{(a+2 L) / \gamma}
$$$$
+2 \beta_{4} e^{(a+L) / \gamma}+24 \gamma^{4} e^{(2 a+L) / \gamma}
$$$$
\left.\left.-2 \beta_{5} \gamma \eta_{1}^{2}+24 \gamma^{4} \eta_{1}\right)\right)
$$$$
C_{3}=\frac{P_{0} e^{-a / \gamma}}{2 \gamma^{2} \operatorname{EI}\left(24 \gamma^{3} \eta_{1}-24 \gamma^{3}+\eta_{3}\left(L^{3}-12 \gamma^{2} L\right)\right)}
$$$$
\times e^{a / \gamma}\left(-a \eta_{3}\left(a^{3}-2 a^{2} L+12 a \gamma^{2}+2 L^{3}\right.\right.
$$$$
\left.-24 \gamma^{2} L\right)+24 \gamma^{4} e^{a / \gamma}
$$$$
\left.+24 a \gamma^{3}+\lambda_{1}+\lambda_{2} e^{L / \gamma}\right)
$$$$
-24 \gamma^{3} \eta_{1}\left(a e^{a / \gamma}-\gamma\right) \text {. }
$$ 


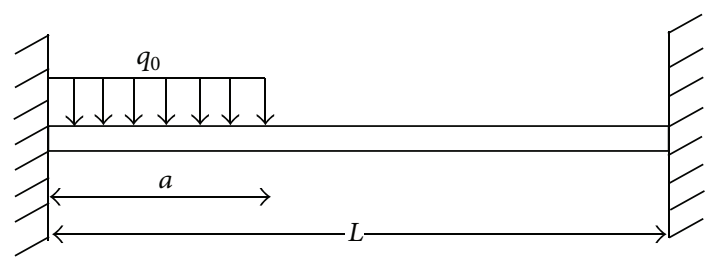

FIGURE 2: Schematic diagram for a microbeam fixed at two ends with discontinuous loading.

Consequently, substituting (24) into (21) and (22), one can get the higher-order deflection functions.

3.2. A Microbeam Subjected to Discontinuous Loading. In this section, it is assumed that the microbeam carries a discontinuous load, as shown in Figure 2. Loading functions are expressed in terms of multiple expressions in different regions (25). Consider

$$
q(z)= \begin{cases}q_{0}, & 0<z<a \\ 0, & a<z<L\end{cases}
$$

This multiple expressions can be simplified by writing a single expression using singularity functions. It is clear that the microbeam is composed of two microbeam segments in the intervals $[0, a]$ and $[a, L]$ so the above equation can be written in terms of Heaviside's unit function as

$$
q(z)=q_{0}\{\vartheta(z)-\vartheta(z-a)\} .
$$

Then, the governing differantial equation is

$$
\mathrm{EI}\left\{\left(\frac{d^{4} v}{d z^{4}}\right)-\gamma^{2}\left(\frac{d^{6} v}{d z^{6}}\right)\right\}=q_{0}\{\vartheta(z)-\vartheta(z-a)\} \text {. }
$$

Taking the Laplace transforms,

$$
\begin{aligned}
& \frac{q_{0}\left(1-e^{-a s}\right)}{\mathrm{EI} s} \\
& =-\gamma^{2}\left(s^{6}\left(\mathscr{L}_{z}[v(z)](s)\right)-v^{(5)}(0)-v^{(4)}(0) s\right. \\
& \left.\quad-v^{(3)}(0) s^{2}-s^{3} v^{\prime \prime}(0)-s^{4} v^{\prime}(0)-v(0) s^{5}\right) \\
& +s^{4}\left(\mathscr{L}_{z}[v(z)](s)\right)-v^{(3)}(0) \\
& -s v^{\prime \prime}(0)-s^{2} v^{\prime}(0)-v(0) s^{3}
\end{aligned}
$$

or

$$
\begin{aligned}
& \mathscr{L}_{z}[v(z)](s)=-\frac{e^{-a s}}{\operatorname{EI} s\left(s^{4}-\gamma^{2} s^{6}\right)} \operatorname{EI} \gamma^{2} s^{6} v(0) e^{a s} \\
& +\mathrm{EI} \gamma^{2} s^{5} e^{a s} v^{\prime}(0)+\mathrm{EI} \gamma^{2} s^{4} e^{a s} v^{\prime \prime}(0) \\
& -\mathrm{EI} s^{4} v(0) e^{a s}+\mathrm{EI} \gamma^{2} s^{3} v^{(3)}(0) e^{a s} \\
& -\mathrm{EI} s^{3} e^{a s} v^{\prime}(0) \gamma^{2} s^{2} v^{(4)}(0) e^{a s} \\
& -\mathrm{EI} s^{2} e^{a s} v^{\prime \prime}(0)+\mathrm{EI} \gamma^{2} s v^{(5)}(0) e^{a s} \\
& -\mathrm{EI} s v^{(3)}(0) e^{a s}-q_{0} e^{a s}+q_{0} .
\end{aligned}
$$

Using the initial conditions in (12),

$$
\begin{aligned}
& \mathscr{L}_{z}[v(z)](s) \\
& =\frac{e^{-a s}\left(e^{a s}\left(\operatorname{EI} s\left(\xi_{1} s\left(\gamma^{2} s^{2}-1\right)+\gamma^{2}\left(\xi_{2} s+\xi_{3}\right)\right)-q_{0}\right)+q_{0}\right)}{\operatorname{EIs}^{5}\left(\gamma^{2} s^{2}-1\right)},
\end{aligned}
$$

where

$$
\xi_{1}=v^{\prime \prime}(0) ; \quad \xi_{2}=v^{(4)}(0) ; \quad \xi_{3}=v^{(5)}(0) .
$$

Similarly, the inverse Laplace transform $\left(\mathscr{L}^{-1}\right)$ of $(30)$ yields

$$
\left.\begin{array}{rl}
\mathscr{L}^{-1}\{v(s)\} & \\
=\mathscr{L}^{-1}\left\{e^{-a s}\right. & \times\left(e ^ { a s } \left(\mathrm{EI} s\left(\xi_{1} s\left(\gamma^{2} s^{2}-1\right)+\gamma^{2}\left(\xi_{2} s+\xi_{3}\right)\right)\right.\right. \\
\left.\left.-q_{0}\right)+q_{0}\right)
\end{array}\right)
$$

where $\vartheta(\cdot)$ is the Heaviside step function. Using the boundary conditions mentioned in the previous section,

$$
\begin{gathered}
\xi_{1}=\frac{\left(m_{1}+m_{2}+m_{3}+m_{4}\right) q_{0}}{12 \mathrm{EIL} m_{5}}, \\
\xi_{2}=\frac{\left(m_{6}-m_{7}+m_{8}\right) q_{0}}{\mathrm{EI} m_{9}}, \\
\xi_{3}=\frac{\left(m_{10}+m_{11}\right) q_{0} e^{L / 2 \gamma}}{\gamma^{2} \mathrm{EI} m_{12}},
\end{gathered}
$$




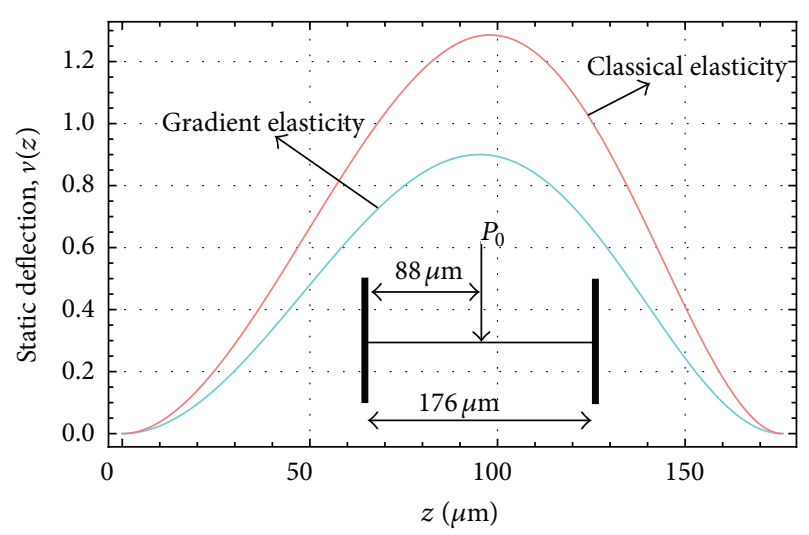

(a)

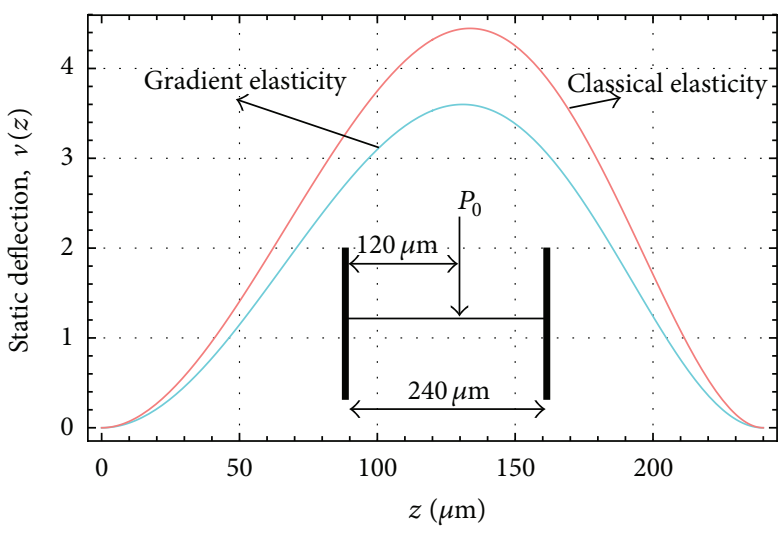

(b)

FIGURE 3: The effect of the material size parameter $\gamma$ on the deflection for $a=88,120 \mu \mathrm{m}$.

where

$$
\begin{aligned}
& m_{1}=6 \gamma\left(a^{3} L(a-2 L)+4 a \gamma^{2}\left(2 a^{2}-3 a L+3 L^{2}\right)-24 \gamma^{4} L\right), \\
& m_{2}=6 \gamma \cosh \left(\frac{L}{\gamma}\right) \\
& \times\left(4 a \gamma^{2}\left(-2 a^{2}+3 a L+3 L^{2}\right)-a L\left(a^{3}-2 a^{2} L+2 L^{3}\right)\right. \\
& \left.+2 \gamma L^{2}\left(L^{2}-6 \gamma^{2}\right) \sinh \left(\frac{a}{\gamma}\right)+24 \gamma^{4} L\right), \\
& m_{3}=L\left(a^{2} L\left(3 a^{2}-8 a L+6 L^{2}\right)\right. \\
& \left.-72 \gamma^{4}(2 a+L)+12 \gamma^{2}(a-L)^{2}(2 a+L)\right) \\
& \times \sinh \left(\frac{L}{\gamma}\right) \text {, } \\
& m_{4}=-12 \gamma^{2} L \cosh \left(\frac{a}{\gamma}\right) \\
& \times\left(L\left(L^{2}-6 \gamma^{2}\right) \sinh \left(\frac{L}{\gamma}\right)+12 \gamma^{3}\left(\cosh \left(\frac{L}{\gamma}\right)-1\right)\right) \\
& -72 \gamma^{4} L \sinh \left(\frac{a}{\gamma}\right)\left(L-2 \gamma \sinh \left(\frac{L}{\gamma}\right)\right), \\
& m_{5}=L\left(L^{2}-12 \gamma^{2}\right) \sinh \left(\frac{L}{\gamma}\right)+24 \gamma^{3}\left(\cosh \left(\frac{L}{\gamma}\right)-1\right) \text {, } \\
& m_{6}=a^{4}-2 a^{3} L+12 a^{2} \gamma^{2}+24 \gamma^{4} \cosh \left(\frac{a}{\gamma}\right)-24 \gamma^{4} \text {, } \\
& m_{7}=\cosh \left(\frac{L}{\gamma}\right)\left(a^{4}-2 a^{3} L+24 \gamma^{4} \cosh \left(\frac{a}{\gamma}\right)\right. \\
& +2 a L^{3}-2 \gamma L\left(L^{2}-12 \gamma^{2}\right) \sinh \left(\frac{a}{\gamma}\right) \\
& \left.+12 a \gamma^{2}(a-2 L)-24 \gamma^{4}\right),
\end{aligned}
$$

$$
\begin{array}{r}
m_{8}=2 \gamma \sinh \left(\frac{L}{\gamma}\right)\left(12 \gamma^{3} \sinh \left(\frac{a}{\gamma}\right)-L\left(L^{2}-12 \gamma^{2}\right)\right. \\
\left.\times \cosh \left(\frac{a}{\gamma}\right)-12 \gamma^{2}(a+L)+L^{3}\right), \\
m_{9}=2 \gamma L\left(L^{2}-12 \gamma^{2}\right) \sinh \left(\frac{L}{\gamma}\right)+48 \gamma^{4}\left(\cosh \left(\frac{L}{\gamma}\right)-1\right), \\
m_{10}=\cosh \left(\frac{L}{2 \gamma}\right)\left(a^{4}-2 a^{3} L-24 \gamma^{4} \cosh \left(\frac{a}{\gamma}\right)\right. \\
\left.+2 a L^{3}+12 a \gamma^{2}(a-2 L)+24 \gamma^{4}\right), \\
m_{11}=24 \gamma^{3}\left(\gamma \sinh \left(\frac{a}{\gamma}\right)+a\right) \sinh \left(\frac{L}{2 \gamma}\right), \\
m_{12}=-24 \gamma^{3}+e^{L / \gamma}\left(24 \gamma^{3}+L^{3}-12 \gamma^{2} L\right)+L^{3}-12 \gamma^{2} L .
\end{array}
$$

\section{Numerical Examples}

In order to demonstrate the efficiency of the present formulation, some illustrative examples are solved and the results are compared with the classical elasticity results. In the numerical results, material scale parameter is taken as $\gamma=$ $17.6 \mu \mathrm{m}$ for homogeneous epoxy beam [8]. These examples demonstrate the usefulness of Laplace transform to the study of discontinuous loading.

4.1. Example 1. In this example, the microbeam have fixed boundary conditions at $(z=0, L)$ and concentrated loading at $(z=a)$, as depicted in Figure 1. For the comparison purposes, the following parameters are used in computing the numerical results: $(a=88,120,176,264),(L=2 a),(\gamma=$ $17.6 \mu \mathrm{m})$, and $\left(P_{0} / \mathrm{EI}=1 / 1000 \mu \mathrm{N} /(\mathrm{GPa} \times \mu \mathrm{m})\right)$. Figures 3,4 , and 5 show that the present deflection values agree very well with the existing data available in the literature. In 


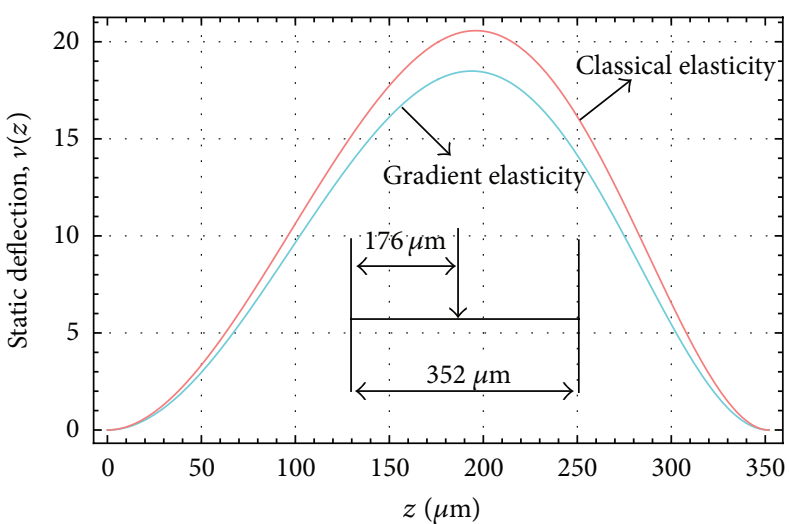

(a)

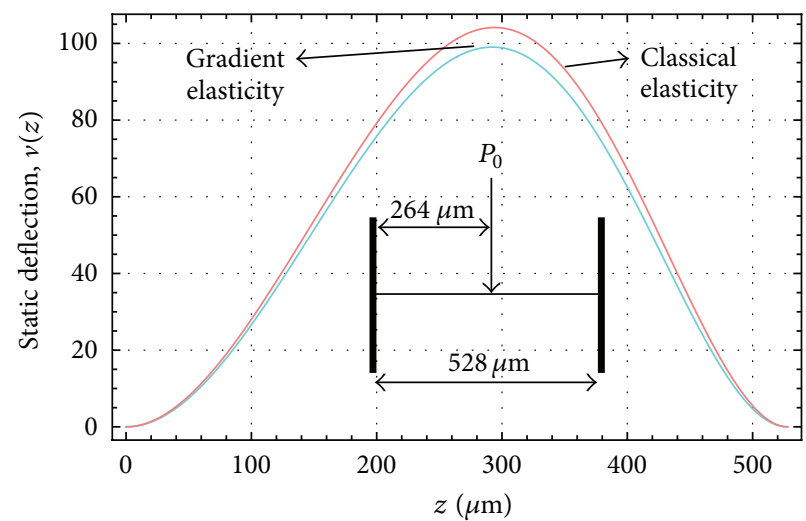

(b)

Figure 4: The effect of the material size parameter $\gamma$ on the deflection for $a=176,264 \mu \mathrm{m}$.
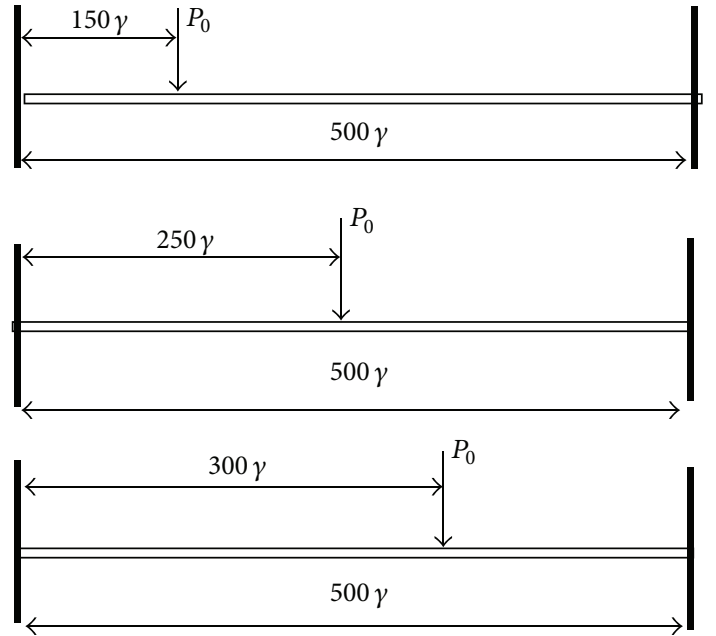

(a)

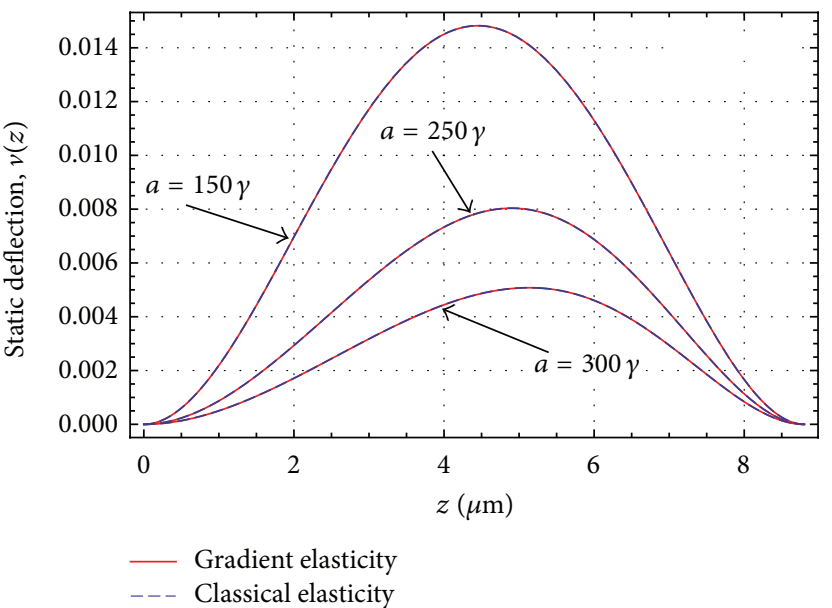

(b)

FIGURE 5: The effect of the material size parameter $\gamma$ on the deflection for $L / \gamma=500, a / \gamma=150,250,300$, and $\gamma=17.6 \mu \mathrm{m}$.

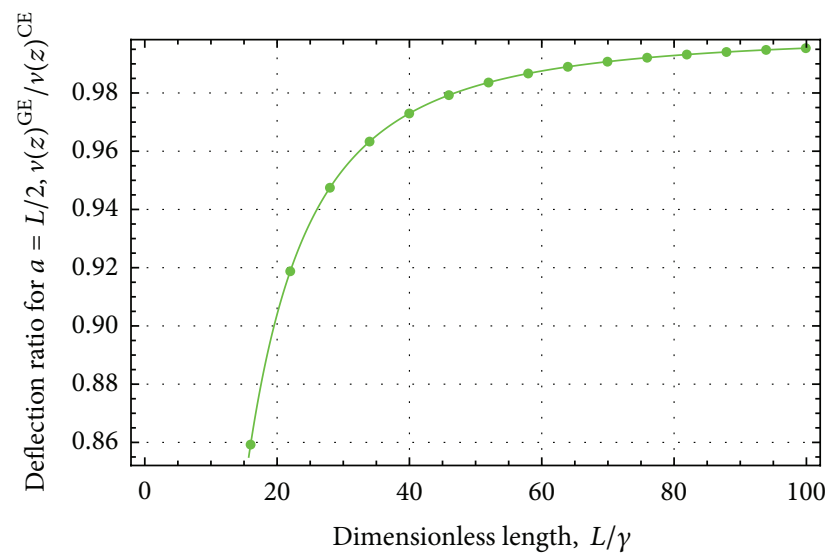

Figure 6: The effect of dimensionless length on the deflection ratios for $L / a=2$ and $\gamma=17.6 \mu \mathrm{m}$.

order to investigate the material size effect, the dimensionless static deflection ratios are also plotted as a function of the dimensionless material parameter in Figure 6. This figure indicates that the strain gradient theory predicts the stiffer behavior for the microbeams compared to the classical theory.

4.2. Example 2. In this example, a microbeam subject to a discontinuous load is considered. For the comparison purposes, the following parameters are used in computing the numerical results: $(a=88 \mu \mathrm{m}, 176 \mu \mathrm{m}, 200 \mu \mathrm{m}, 240 \mu \mathrm{m})$, $(L=2 a),(\gamma=17.6 \mu \mathrm{m}),\left(q_{0} / \mathrm{EI}=1 / 1000 \mu \mathrm{N} /\left(\mathrm{GPa} \times \mu \mathrm{m}^{2}\right)\right)$. Figures 7 and 8 display the effect of material parameter on the static behavior of the microbeam. It is clear that material parameter has a significant effect on the static deflection. Present results are in consistency with that for the gradient elastic model in [18].

It can be noted that the results predicted by the gradient elasticity theory are always smaller than those of the classical beam theory. Further, the difference between the two results is remarkable for the microbeams with $L / \gamma \leq 10$. It is wellknown in the literature that gradient elasticity (the modified couple stress theory) evaluates the microbeams stiffer than evaluations of the classical theory. 


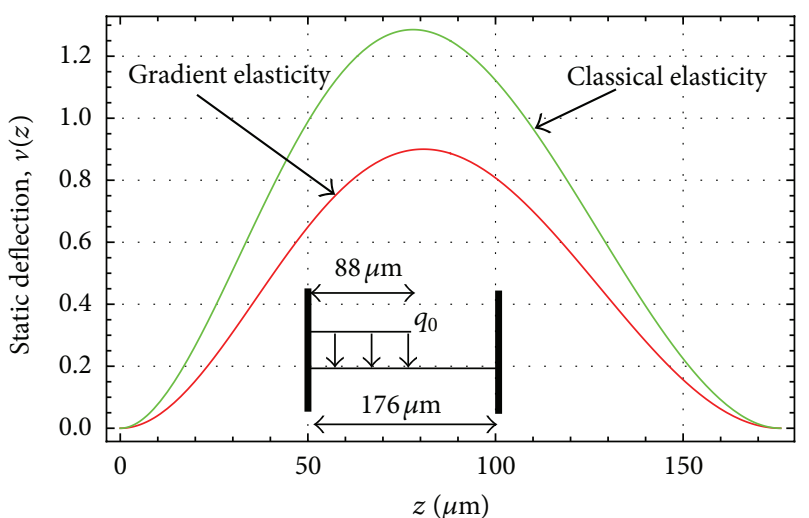

(a)

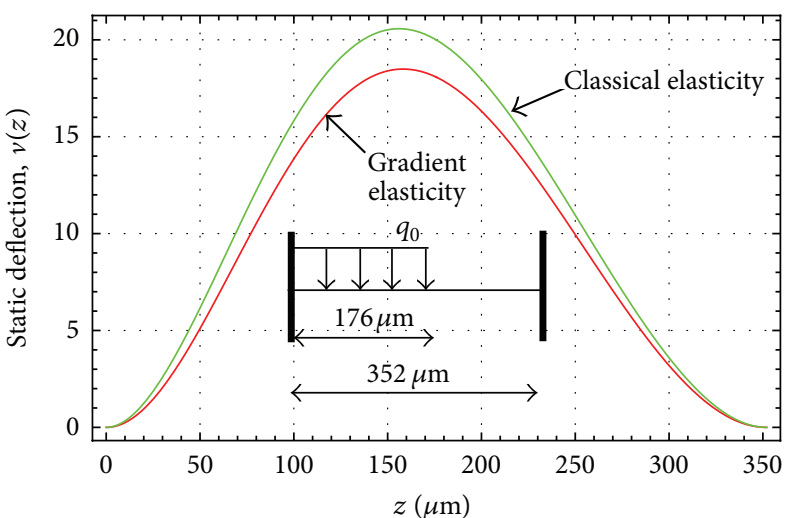

(b)

FIgURE 7: The effect of the material size parameter $\gamma$ on the deflection for $a=88,176 \mu \mathrm{m}$.

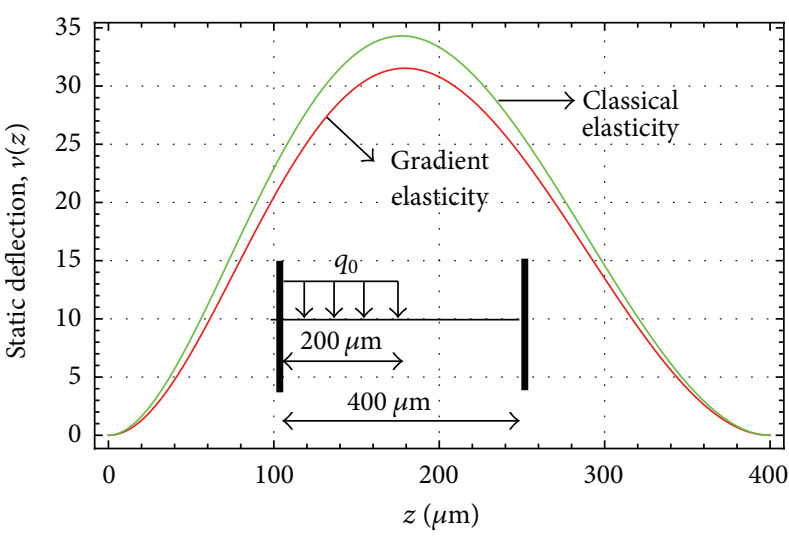

(a)

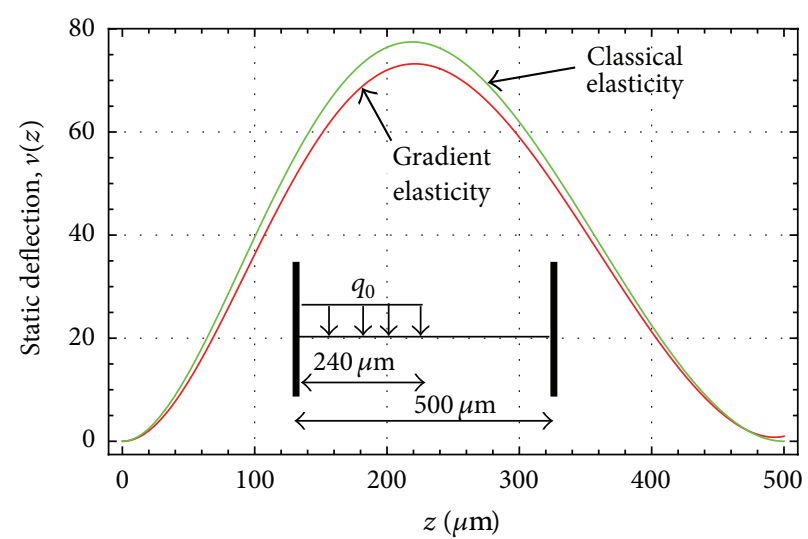

(b)

FIGURE 8: The effect of the material size parameter $\gamma$ on the deflection for $a=200,240 \mu \mathrm{m}$.

\section{Conclusion}

Based on strain gradient elasticity theory with surface energy, the static bending solutions of the gradient elastic beams with external discontinuities are presented by Laplace transform. Explicit expressions are derived for static deflections for clamped-clamped boundary conditions. Derived expressions are verified for several numerical examples and the results show that the Laplace transform gives reliable results. Comparison of classical elasticity theory also performed to verify the present solutions. It can be concluded that the difference between the results predicted by the classical beam theories and those evaluated by the strain gradient theories are significant when $L / \gamma$ is low. The main contribution of this paper is to provide a closed-form solution for the static deflection of microbeams under geometric discontinuities.

\section{Conflict of Interests}

The author declare that there is no conflict of interests regarding the publication of this paper.

\section{References}

[1] E. S. Hung and S. D. Senturia, "Extending the travel range of analog-tuned electrostatic actuators," Journal of Microelectromechanical Systems, vol. 8, no. 4, pp. 497-505, 1999.

[2] P. Attia, G. Tremblay, R. Laval, and P. Hesto, "Characterisation of a low-voltage actuated gold microswitch," Materials Science and Engineering B, vol. 51, no. 1-3, pp. 263-266, 1998.

[3] H. A. C. Tilmans and R. Legtenberg, "Electrostatically driven vacuum-encapsulated polysilicon resonators. Part II. Theory and performance," Sensors and Actuators A, vol. 45, no. 1, pp. 67-84, 1994.

[4] M. H. Kahrobaiyan, M. T. Ahmadian, P. Haghighi, and A. Haghighi, "Sensitivity and resonant frequency of an AFM with sidewall and top-surface probes for both flexural and torsional modes," International Journal of Mechanical Sciences, vol. 52, no. 10, pp. 1357-1365, 2010.

[5] Y. Moser and M. A. M. Gijs, "Miniaturized flexible temperature sensor," Journal of Microelectromechanical Systems, vol. 16, no. 6, pp. 1349-1354, 2007.

[6] S. M. Cook, T. E. Schäffer, K. M. Chynoweth, M. Wigton, R. W. Simmonds, and K. M. Lang, "Practical implementation of dynamic methods for measuring atomic force microscope 
cantilever spring constants," Nanotechnology, vol. 17, no. 9, pp. 2135-2145, 2006.

[7] N. A. Fleck, G. M. Muller, M. F. Ashby, and J. W. Hutchinson, "Strain gradient plasticity: theory and experiment," Acta Metallurgica et Materialia, vol. 42, no. 2, pp. 475-487, 1994.

[8] D. C. C. Lam, F. Yang, A. C. M. Chong, J. Wang, and P. Tong, "Experiments and theory in strain gradient elasticity," Journal of the Mechanics and Physics of Solids, vol. 51, no. 8, pp. 14771508, 2003.

[9] H. M. Ma, X.-L. Gao, and J. N. Reddy, "A microstructure-dependent Timoshenko beam model based on a modified couple stress theory," Journal of the Mechanics and Physics of Solids, vol. 56, no. 12, pp. 3379-3391, 2008.

[10] G. C. Tsiatas, "A new Kirchhoff plate model based on a modified couple stress theory," International Journal of Solids and Structures, vol. 46, no. 13, pp. 2757-2764, 2009.

[11] B. Arash and Q. Wang, "Stability analysis of nonlocal elastic columns with initial imperpection," Compuatational Materials Science, vol. 51, no. 1, pp. 303-313, 2012.

[12] S. P. Xu, M. R. Xu, and C. M. Wang, "Stability analysis of nonlocal elastic columns with initial imperfection," Mathematical Problems in Engineering, vol. 2013, Article ID 341232, 12 pages, 2013.

[13] M. O. Yayli, "Torsion of nonlocal bars with equilateral cross sections," Journal of Computational and Theoretical Nanoscience, vol. 10, no. 2, pp. 376-379, 2013.

[14] F. Yang, A. C. M. Chong, D. C. C. Lam, and P. Tong, "Couple stress based strain gradient theory for elasticity," International Journal of Solids and Structures, vol. 39, no. 10, pp. 2731-2743, 2002.

[15] S. K. Park and X.-L. Gao, "Bernoulli-Euler beam model based on a modified couple stress theory," Journal of Micromechanics and Microengineering, vol. 16, no. 11, pp. 2355-2359, 2006.

[16] M. Şimşek, "Dynamic analysis of an embedded microbeam carrying a moving microparticle based on the modified couple stress theory," International Journal of Engineering Science, vol. 48, no. 12, pp. 1721-1732, 2010.

[17] M. Ö. Yayli, "Weak formulation of finite element method for nonlocal beams using additional boundary conditions," Journal of Computational and Theoretical Nanoscience, vol. 8, no. 11, pp. 2173-2180, 2011.

[18] S. Papargyri-Beskou, K. G. Tsepoura, D. Polyzos, and D. E. Beskos, "Bending and stability analysis of gradient elastic beams," International Journal of Solids and Structures, vol. 40, no. 2, pp. 385-400, 2003.

[19] S. Papargyri-Beskou and D. E. Beskos, "Response of gradientviscoelastic bar to static and dynamic axial load," Acta Mechanica, vol. 170, no. 3-4, pp. 199-212, 2004. 


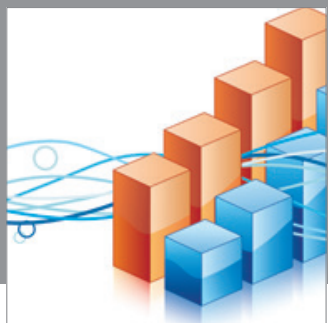

Advances in

Operations Research

mansans

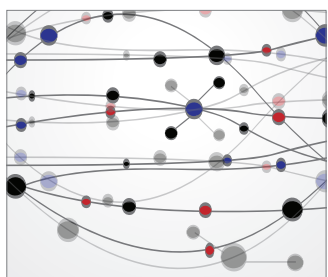

The Scientific World Journal
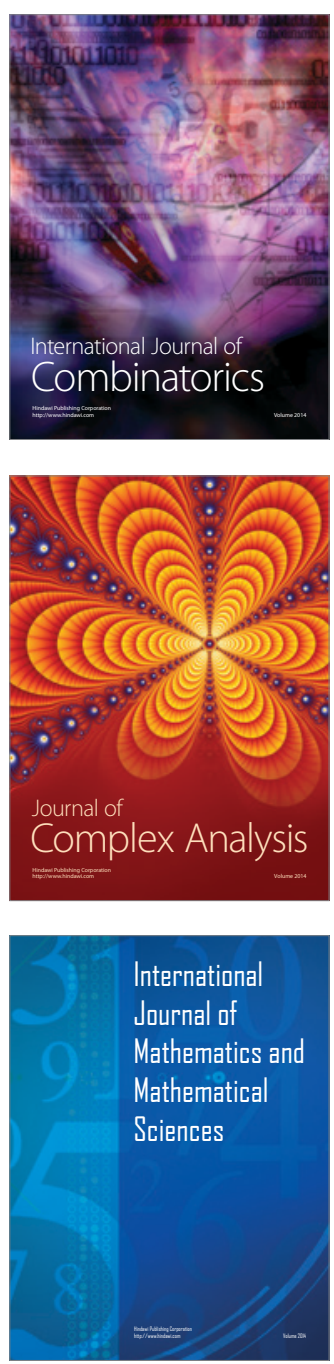
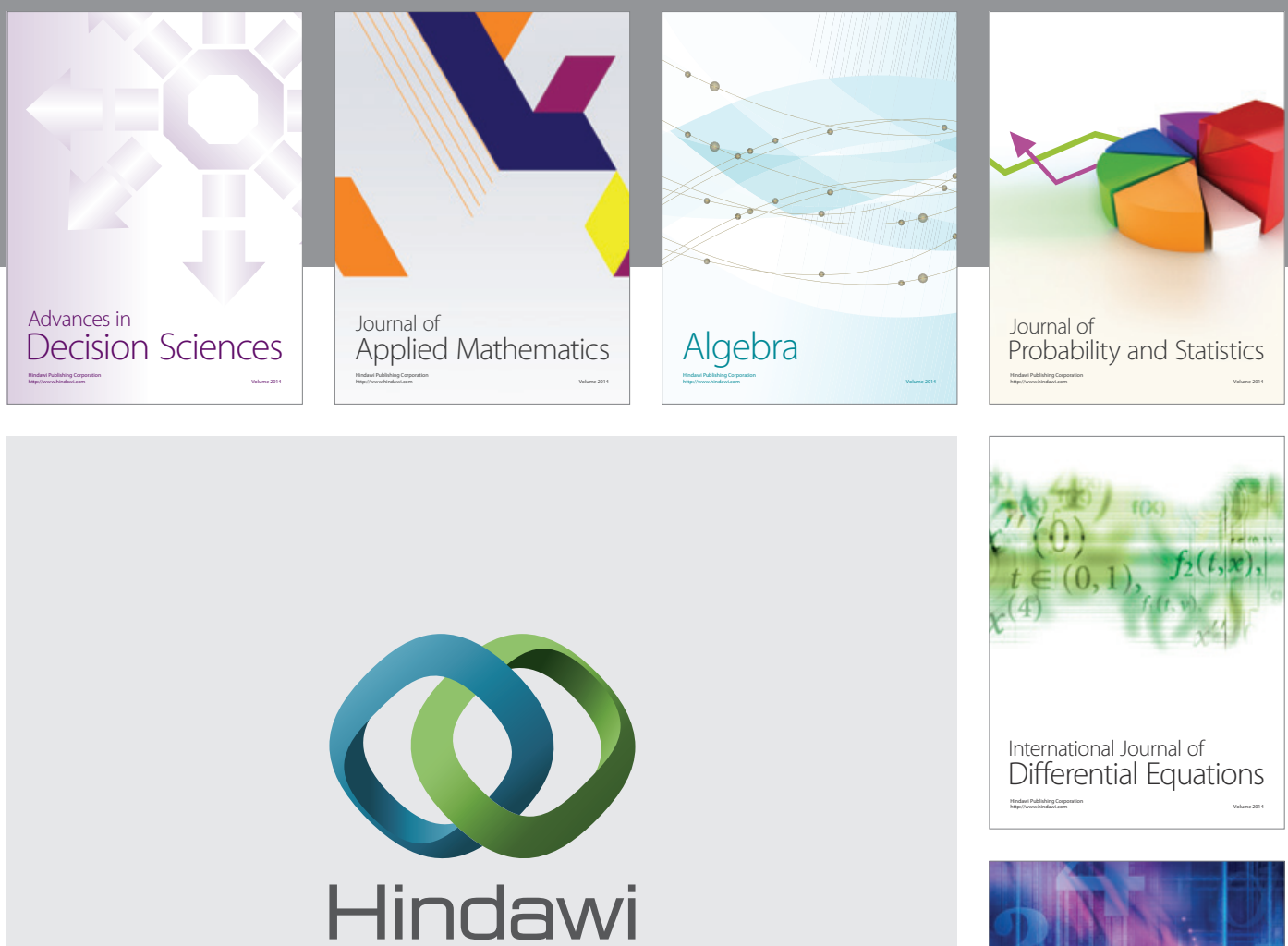

Submit your manuscripts at http://www.hindawi.com
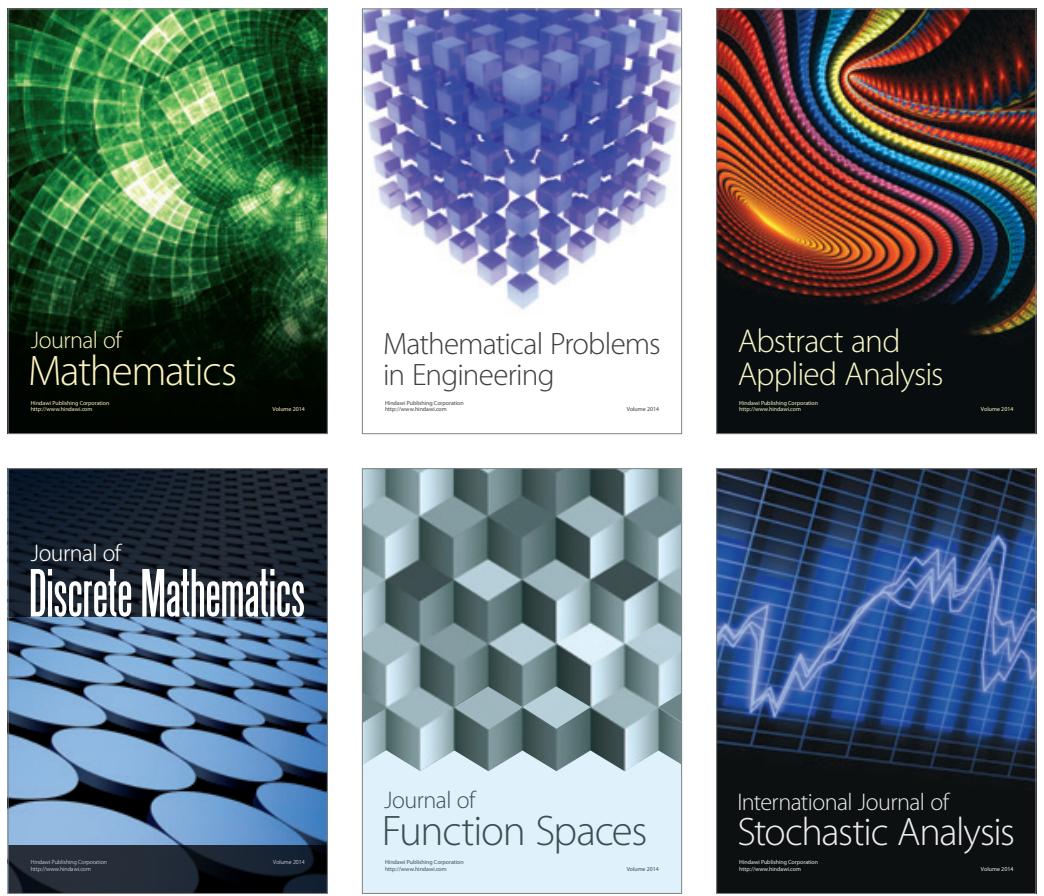

Journal of

Function Spaces

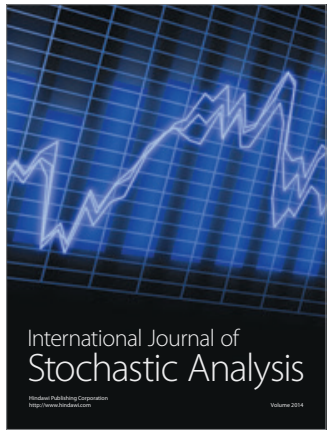

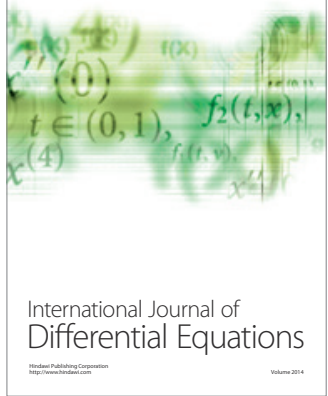
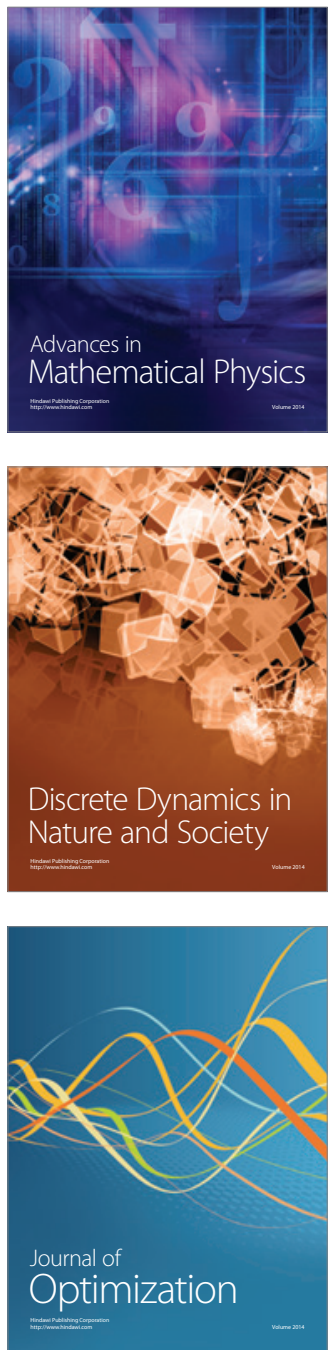